\title{
Imaging at High Spatial Resolution: Soft X-Ray Microscopy to $15 \mathrm{~nm}$
}

\author{
David Attwood*\#, Weilun Chao*\#, Erik Anderson", J. Alexander Liddle ${ }^{\#}$, Bruce Harteneck ${ }^{\#}$, \\ Peter Fischer $^{\#}$, Gerd Schneider ${ }^{\S}$, Mark Le Gros ${ }^{\ddagger}$ and Carolyn Larabell ${ }^{\dagger \neq}$ \\ *University of California, Berkeley, CA USA \\ \#Center for X-Ray Optics, Lawrence Berkeley National Laboratory, Berkeley, CA USA \\ $\S B E S S Y$ Synchrotron Facility, Berlin, Germany
}

$\ddagger$ National Center for X-Ray Tomography, Lawrence Berkeley National Laboratory, Berkeley

$†$ Department of Anatomy, University of California, San Francisco, CA USA

Corresponding details (e-mail: attwood@eecs.berkeley.edu, tel: 510-486-4463, fax: 510-

486-4955)

Date of submission ()

\begin{abstract}
:
Soft x-ray microscopy has now achieved $15 \mathrm{~nm}$ spatial resolution with new zone plates and bending magnet radiation. Combined with elemental sensitivity and flexible sample environment (applied magnetic or electric fields, wet samples, windows, overcoatings) this emerges as a valuable tool for nanoscience and nanotechnology, complimenting common electron and scanning tip microscopies. In this presentation we describe recent advances in spatial resolution, expectations for the near future, and applications to magnetic materials, bio-tomography, etc.
\end{abstract}




\section{Key Words:}

\section{The Soft X-ray spectral region}

The soft $\mathrm{x}$-ray spectral region offers several very interesting features ${ }^{1}$. The photon energies in this region, typically $250 \mathrm{eV}$ to several $\mathrm{KeV}$, match very well to the atomic resonances of almost all elements, thus permitting very clear elemental and even chemical identification when probing material. Furthermore, the wavelengths in this region are very short, typically one to a few nanometers, in principle permitting high resolution imaging. The soft $\mathrm{x}$-ray spectral region is identified in Figure 1. In addition to clear elemental discrimination, proper use of the photon energy can be used to image wet objects in several micron thick water, important for biological and environmental studies, and also to look through substrates or capping layers in electronic or magnetic structures. Furthermore, there is a natural magnetic contrast in this spectral region, with circularly (or elliptically) polarized radiation, due to differences in spin-orbit coupling among the initial and final states of elements such as iron, cobalt, etc. The combination of all these attributes: elemental and chemical identification, wet or overcoated samples, polarization sensitive absorption, and short wavelengths for potential high resolution imaging, all come to bear in the development and application of soft x-ray microscopy ${ }^{2}$. 


\section{Soft X-ray microscopy}

High spatial resolution soft x-ray microscopy has been achieved with Fresnel zone plate lenses, essentially circular diffraction gratings with the zones positioned to give positive interference at a well defined focal spot, or point by point within an image ${ }^{1}$. Figure 2 shows an image of one quadrant of a circular zone plate having an outer zone width ( r) of $25 \mathrm{~nm}$, a diameter of $63 \mu \mathrm{m}$, and a total of 618 zones $^{3}$. A diagram of the full field microscope, XM-1, at the Advanced Light Source (ALS) in Berkeley, is shown in Figure 3. A test pattern image of $15 \mathrm{~nm}$ wide $\mathrm{Cr}$ and $\mathrm{Si}$ lines, obtained with a new zone plate lenses ${ }^{4}$, having $15 \mathrm{~nm}$ outer zone width, is shown in Figure 4. The image was obtained at a wavelength of $1.52 \mathrm{~nm}$. Examples of images for the life and physical sciences are shown in Figure 5. The first shows the image of an FeTbCo multilayer with an Al capping layer. The image was obtained by P. Fischer and his colleagues ${ }^{5}$ with a $35 \mathrm{~nm}$ zone plate, just at the $\mathrm{Fe}_{3}$ edge $(707.5 \mathrm{eV}, 1.75 \mathrm{~nm})$. This form of microscopy has great advantages for the study of magnetic materials: high spatial resolution approaching the fundamental limit set by grain size, tunability to the absorption edges of specific elements which then dominate the image, tunability between $\mathrm{L}_{2}$ and $\mathrm{L}_{3}$ edges for the observation of spin-orbit coupling effects, magnetic fields can be applied and changed during imaging, in-plane and out-of-plane measurements are possible, thin capping layers and substrates are possible.

The second and third images in Figure 5 show examples of biological material imaged with soft 
X-rays at XM-1, both obtained by Carolyn Larabell and her colleagues ${ }^{6}$. The center image is that of full thickness, hydrated and unstained fibroblast cells obtained with natural contrast in the water window at $520 \mathrm{eV}$, just below the oxygen $\mathrm{K}$-edge, at $2.4 \mathrm{~nm}$ wavelength. The sample has been cryo-fixed by quick freezing, which permits more than 100 images to be obtained without noticeable effect on the finest features. For the third image, also obtained at $2.4 \mathrm{~nm}$ wavelength, labeling has been used to show the location of specific proteins, forming a microtubule network in a fully hydrated, unsectioned, mouse epithelial cell. The label consists of highly absorbing $\mathrm{Au} / \mathrm{Ag}$ nanoparticles, about equal in size to the spatial resolution of the microscope. Such labels play an important role for biologists who wish to study quantitative distributions of specific proteins as a function of cell cycle, gene availability and functionality.

Several approaches to three-dimensional imaging have been proposed. The top diagram in Figure 6 shows the technique ${ }^{7}$ employed at XM-1 in which samples are rotated in a thin capillary tube, obtaining sequential images, which are then deconvolved using computer, aided tomography (CAT scan) software to reconstruct full three-dimensional images. At the lower left in Figure 6 is an example of an image ${ }^{8}$ at $2.5 \mathrm{~nm}$ wavelength of a yeast cell, one of 45 images obtained at $4^{\circ}$ intervals, for a total coverage of $180^{\circ}$. The reconstructed image ${ }^{8}$ of a single yeast cell showing the cell nucleus (purple), an enlarged vacuole, many lipid filled vesicles, and details near the outer cell wall, is shown at the lower right. Future plans call for a new soft x-ray microscope, 
XM-2 at the ALS, dedicated to tomographic imaging of biological samples making significant use of protein labeling to study the quantitative distribution of specific proteins in healthy and genetically impaired cells 9 .

\section{Conclusion:}

Soft x-ray microscopy is coming of age. As a new technique for studying nanoscale materials, it compliments existing techniques such as visible light, electron and scanning tip microscopies. Soft x-ray microscopy offers unique advantages with regard to elemental and chemical identifications, spatial resolution soon to reach $10 \mathrm{~nm}$, polarization control for the study of magnetic and chiral structures, the ability to see through thin capping layers and substrates, and use in the presence of time variable electric and magnetic fields. Its downside to date is the need for a large synchrotron radiation facility, albeit only a bending magnet for the full field microscope at modest spectral resolution $(\sim \sim 700)$. There are, however, about thirty such facilities worldwide, each with many bending magnets. Perhaps in the not too distant future more compact laser-based sources (laser-plasma, SXR laser [10], or SXR HHG [11]) will become available for use in advanced university and industrial research labs.

\section{References:}

[1] Attwood, D. Soft X-Rays and Extreme Ultraviolet Radiation (Cambridge University Press, Cambridge, 2000). 
[2] Aoki, S. et al., XRM-2005 Proceedings (X-Ray Microscopy Conference, Himeji, Japan, July 2005).

[3] Chao, W.L. et al. $20 \mathrm{~nm}$ Resolution Soft X-Ray Microscopy Demonstrated by use of Multilayer Test Structures. Optics Lett. 28, 2019 (2003).

[4] Chao, W.L. et al. Soft X-Ray Microscopy at a Spatial Resolution Better Than $15 \mathrm{~nm}$, Nature. 435, 1210 (30 June 2005).

[5] Fischer, P. et al. High Resolution Imaging of Magnetic Domains with Magnetic Soft X-Ray Microscopy, J. Magn. Soc. of Japan. 25, 186 (2001); Fischer, P. et al. Study of Magnetic Domains by Magnetic Soft X-Ray Transmission Microscopy, J. Phys. D (London). 35, 2391 (2002).

[6] Meyer-Ilse, W. et al. High Resolution Protein Localization using Soft X-Ray Microscopy, J. Microsc. 201, 395 (2001).

[7] Schneider, G. et al. Computed Tomography of Cryogenic Cells, Surf. Rev. Lett. 9, 177 (2002).

[8] Larabell, C. and Gros, M.Le. X-Ray Tomography Generates 3-D Reconstruction of the Yeast, Saccharomyces Cerevisiae, at 60 nm Resolution, Mol. Biol. Cell. 15, 957 (2003).

[9] C. Larabell, personal communication

[10] Larotonda, M. et al. Characteristics of a Saturated $18.9 \mathrm{~nm}$ Table Top Laser Operating at 5 Hz Repetition Rate, IEEE J. Select. Topics Quant. Electron. 10, 1363 (2004). 
[11] Gibson, E. et al. Coherent Soft X-Ray Generation in the Water Window with Quasi-Phase Matching, Science 302, 95 (2003).

\section{Figure Caption:}

Figure 1. The Soft x-ray region of the electromagnetic spectrum is particularly useful for studying physical and biological systems with elemental, chemical, and magnetic sensitivity at high spatial resolution.

Figure 2. A Fresnel zone plate lens for soft x-ray microscopy, with 618 zones, $25 \mathrm{~nm}$ outer zone width, and diameter of 63 microns. Typical wavelength use is from 1 to $4 \mathrm{~nm}$.

Figure 3. The full field soft x-ray microscope XM-1 uses bending magnet radiation at the ALS. Exposure times are typically one to a few seconds for a wide range of applications including magnetic materials, cryo-fixed biological samples, wet environmental samples, etc. Mutual indexing with a visible light microscope permits very efficient use, often resulting in several hundred to one thousand images per day.

Figure 4. Soft x-ray image of a pattern of $15 \mathrm{~nm}$ wide lines of $\mathrm{Cr}$ and $\mathrm{Si}$, obtained at $1.52 \mathrm{~nm}$ wavelength with the XM-1 microscope at the ALS (W.L. Chao et al., Nature, 2005).

Figure 5. Soft x-ray images of a magnetic structure; a natural contrast, unsectioned, fully hydrated fibroblast cell; a protein labeled microtubulen network in a mouse epithelial cell. The first image (left side) is of an FeTbCo magnetic multilayer, with an Al overlayer, obtained at the 
Fe $\mathrm{L}_{3}$ edge at $707.5 \mathrm{eV}(1.75 \mathrm{~nm})$. The second image (center) is obtained at $520 \mathrm{eV}$ in the water window, at a wavelength of $2.4 \mathrm{~nm}$. The third image (right) was also obtained at $520 \mathrm{eV}$. (See references to P. Fischer, W. Meyer-Ilse and C. Larabell).

Figure 6. Computer aided tomography (3-D CAT scans) of cryo-fixed yeast cells is obtained by rotating the cells in a thin walled capillary (top) while taking sequential images at $2.5 \mathrm{~nm}$ in the so-called "water window" where carbon containing structures absorb soft x-rays but water is relatively transparent to thicknesses of 5-10 microns. The lower left image shows one of 45 images, taken at $4^{\circ}$ angular intervals, of a yeast cell. The lower right image shows a tomographic reconstruction of the yeast cell at approximately $60 \mathrm{~nm}$ spatial resolution. Future images will be taken at $1^{\circ}$ angular intervals and higher resultant spatial resolution. (C. Larabell and M. Le Gros [8]). 


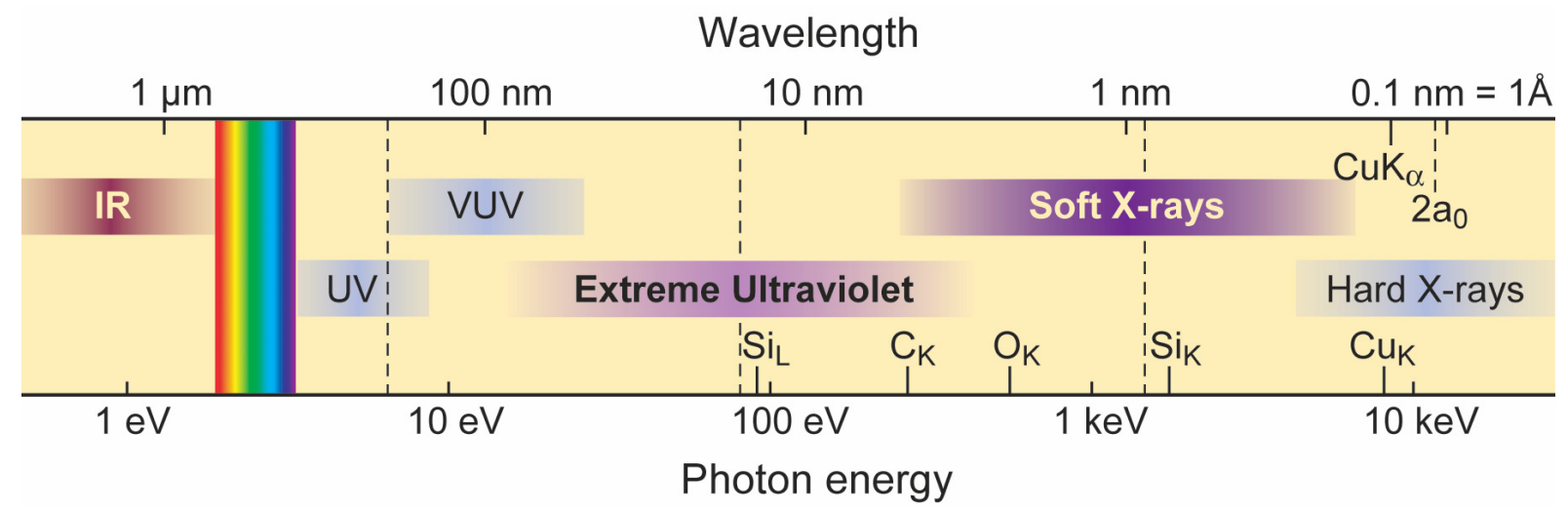

Figure 1 


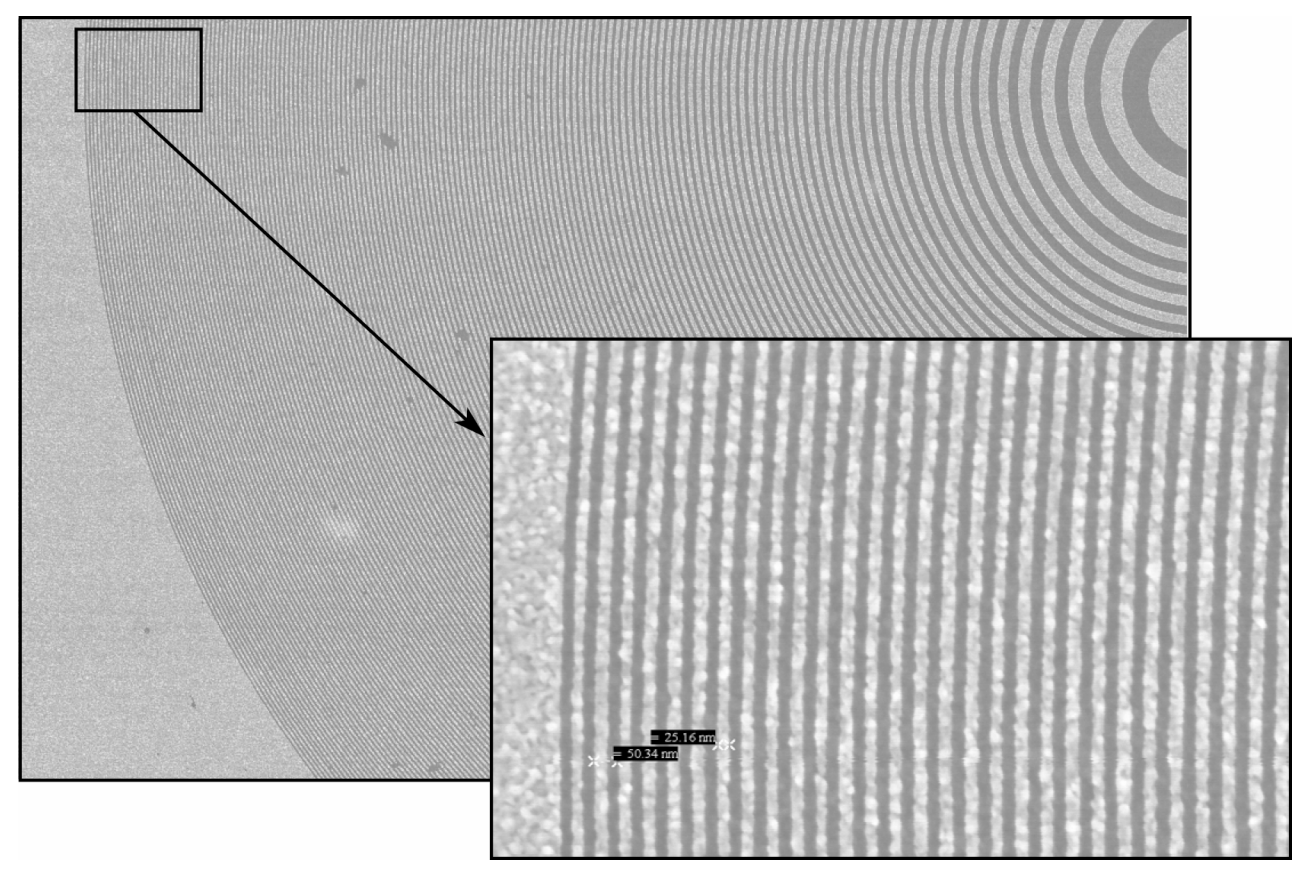

Figure 2 


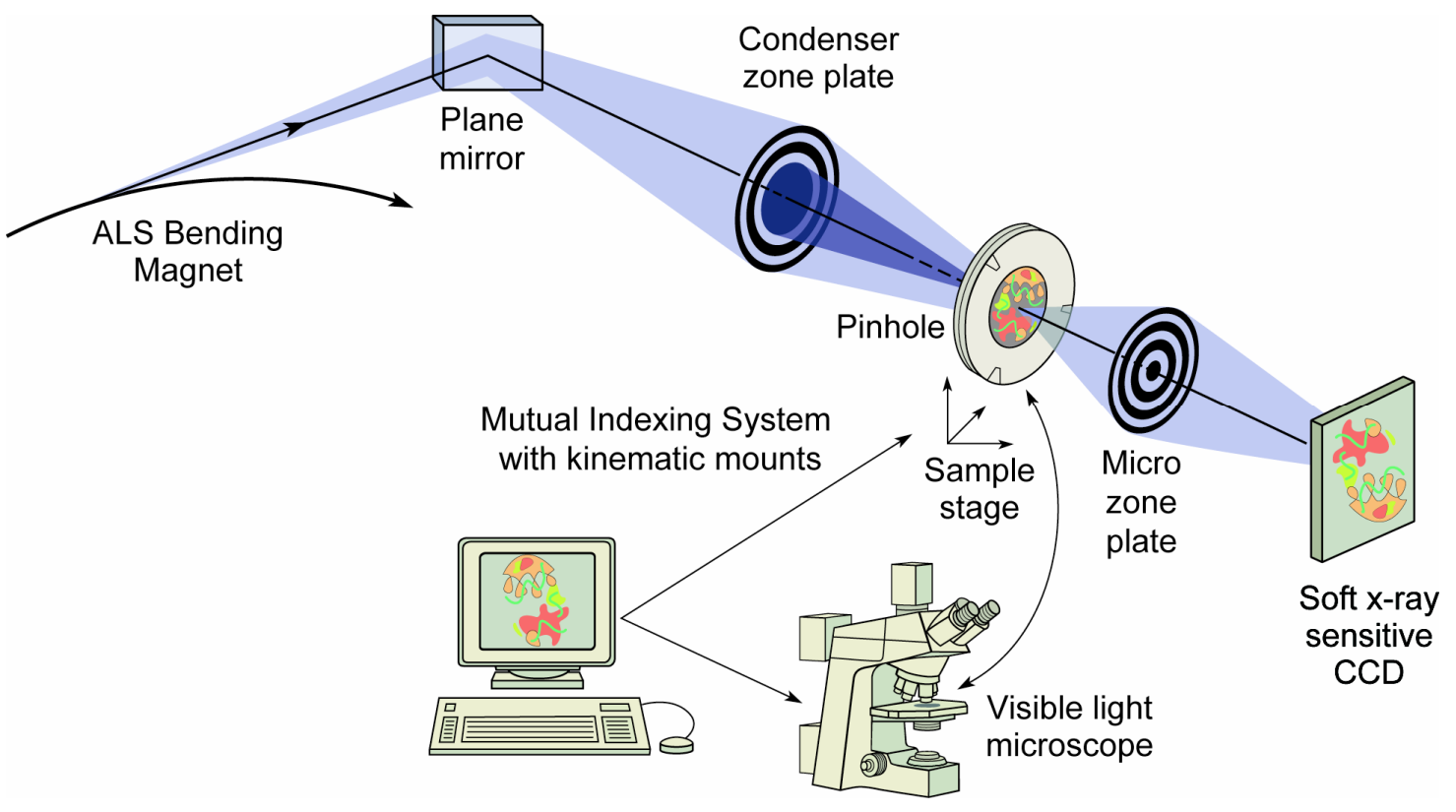

Figure 3 


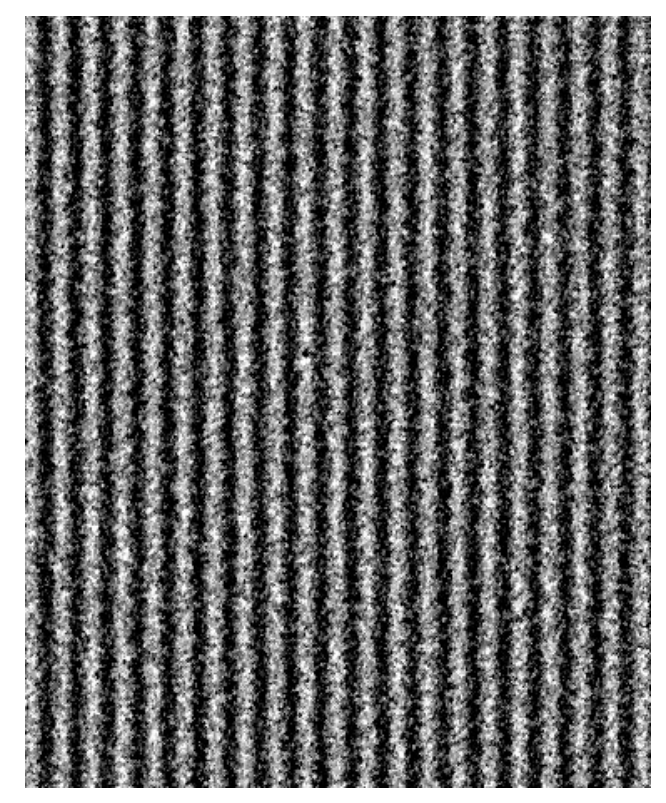

Figure 4 


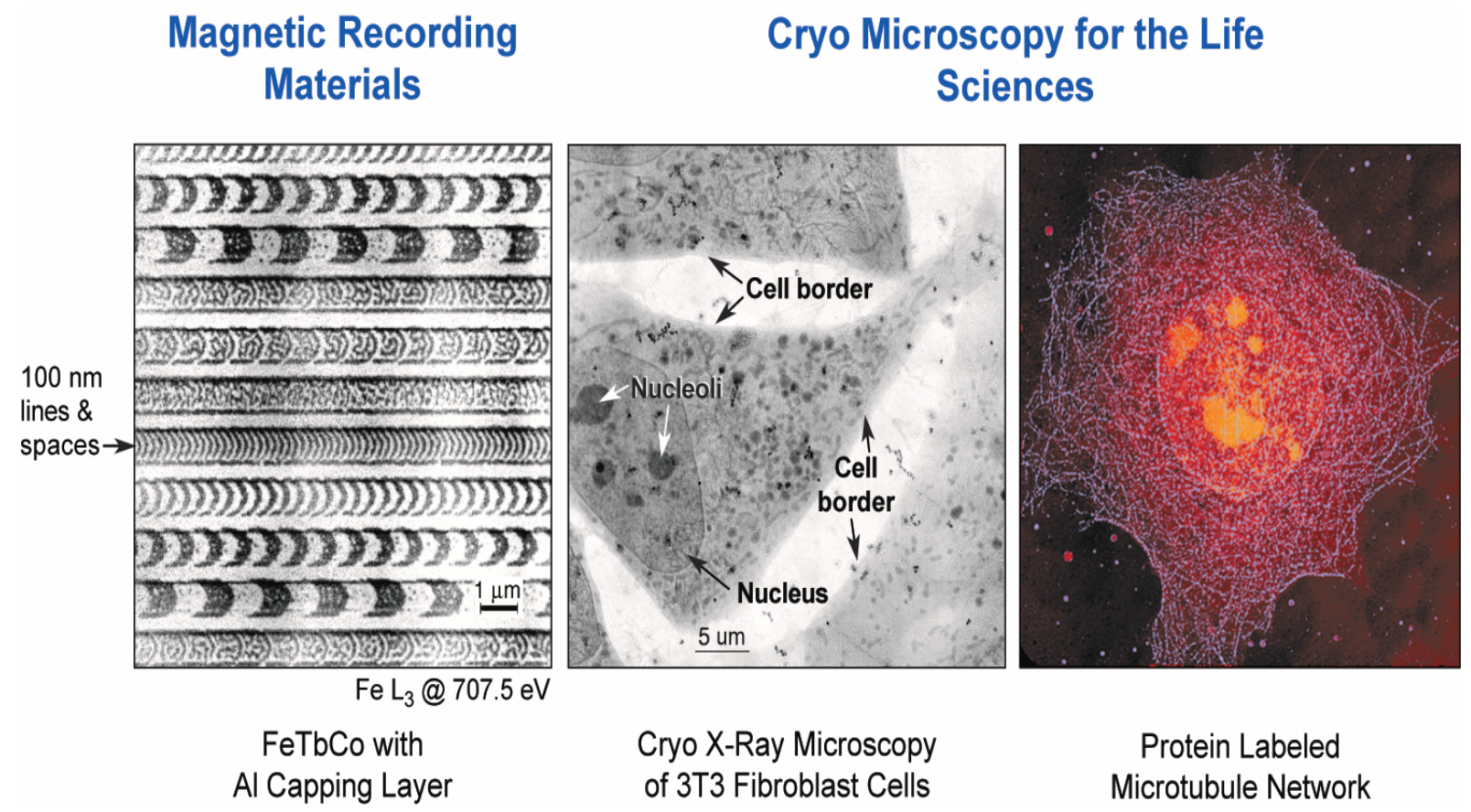

Figure 5 


\section{Nanotomography of Cryogenic Fixed Cells}

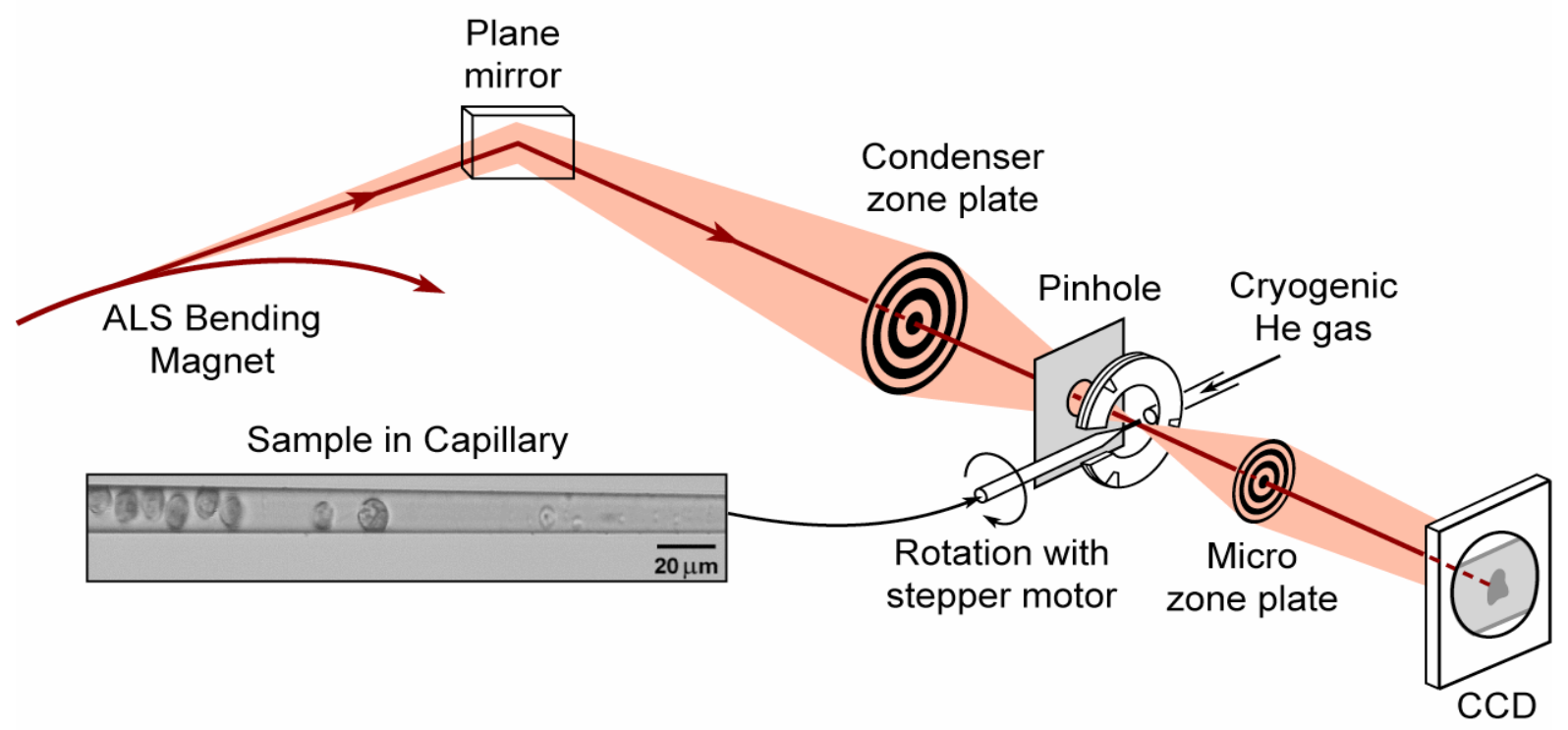

Soft X-Ray Nanotomograpy of a Yeast Cell
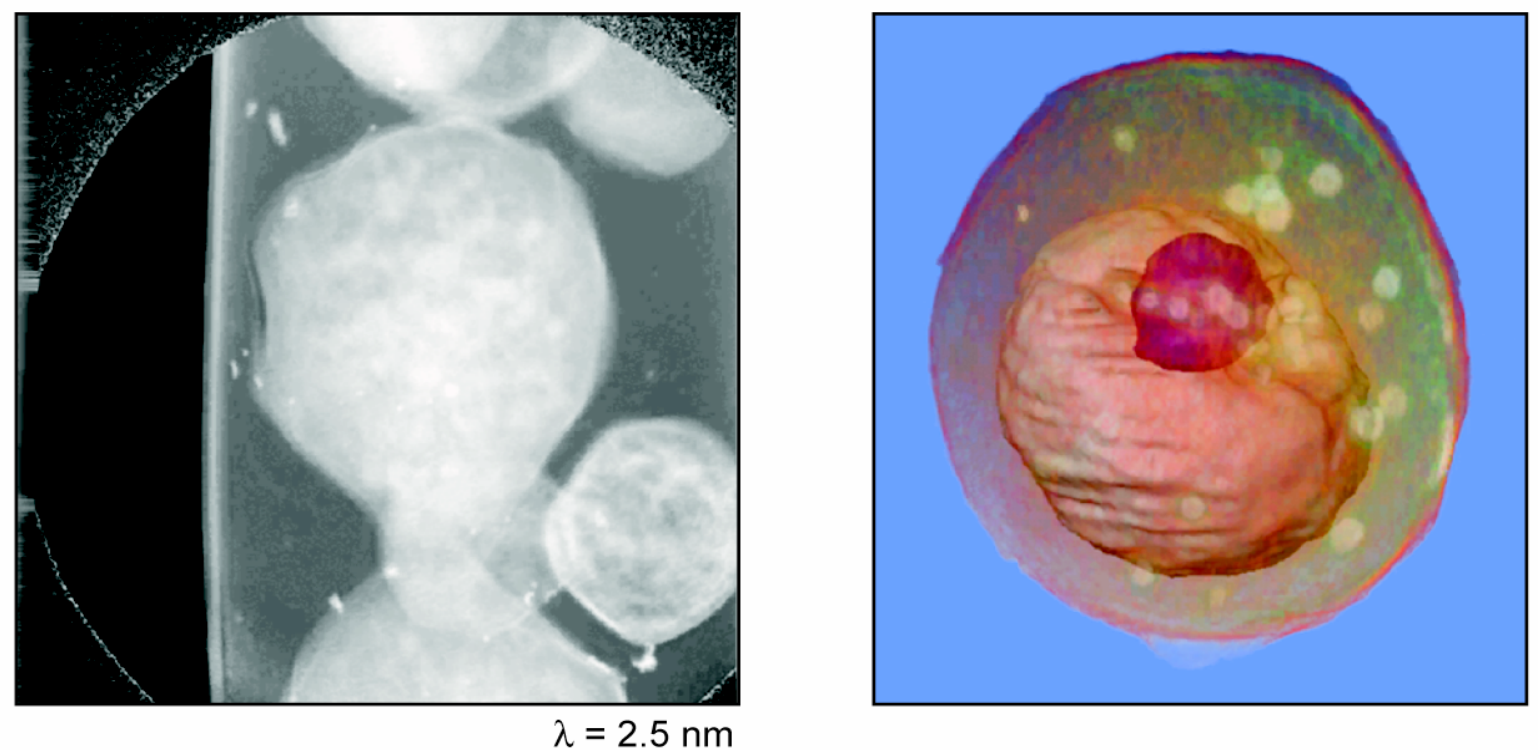

Figure 6 